\title{
Micromagnetic Investigation of Periodic Cross-Tie/Vortex Wall Geometry
}

\author{
Michael J. Donahue \\ Applied and Computational Mathematics Division, National Institute of Standards and Technology, Gaithersburg, \\ MD 20899-8910, USA \\ Correspondence should be addressed to Michael J. Donahue, michael.donahue@nist.gov
}

Received 31 March 2012; Accepted 18 May 2012

Academic Editor: Eduardo Martinez Vecino

Copyright (C) 2012 Michael J. Donahue. This is an open access article distributed under the Creative Commons Attribution License, which permits unrestricted use, distribution, and reproduction in any medium, provided the original work is properly cited.

\begin{abstract}
A systematic series of micromagnetic simulations on periodic cross-tie/vortex wall structures in an ideal soft film at various widths, thicknesses, and period lengths is performed. For each width and thickness a natural period length is found which has minimal energy density for walls of this type. For each width, a critical thickness is determined below which the natural period length is infinite; for films thinner than this, the pure Néel wall has lower energy than any cross-tie/vortex wall. Details of the origin of the energy reduction in cross-tie/vortex walls as compared to Néel walls are also examined, and canting inside cross-tie and vortex structures in films thicker than $1 \ell_{\text {ex }}$ is explained.
\end{abstract}

\section{Introduction}

The predominant types of $180^{\circ}$ domain walls in soft films are Néel walls occurring in very thin films, Bloch walls in thicker samples, and numerous transitional structures [1-3]. One of the more interesting transitional structures is the crosstie/vortex (or simply cross-tie) wall which alternates crossties and vortices between counterrotating segments of Néel walls. Cross-tie/vortex walls are observed experimentally [4-6], in micromagnetic simulations [6-9], and in theory $[10,11]$. In finite samples, this wall type appears in low remanence closed-flux Landau patterns, as seen in Figure 1. If the structure is long enough, then multiple cross-ti/evortex pairs can appear, as in Figure 2.

To gain insight into the formation and structure of crosstie/vortex walls, this work presents a systematic collection of micromagnetic simulations performed using the OOMMF micromagnetic package from NIST [12]. An ideally soft material was modeled (anisotropy constant $K=0 \mathrm{~J} / \mathrm{m}^{3}$ ) with saturation magnetization $M_{\mathrm{s}}=860 \mathrm{kA} / \mathrm{m}$ and exchange coefficient $A=13 \mathrm{pJ} / \mathrm{m}$, to approximate an NiFe alloy. All of the simulations were performed with no applied field.
The component energies in this system are the stray field energy and the exchange energy, with the component fields defined by:

$$
\begin{aligned}
& \mathbf{H}_{\text {stray field }}(\mathbf{r})=-\frac{1}{4 \pi} \int_{V} \nabla \cdot \mathbf{M}\left(\mathbf{r}^{\prime}\right) \frac{\mathbf{r}-\mathbf{r}^{\prime}}{\left|\mathbf{r}-\mathbf{r}^{\prime}\right|^{3}} d^{3} r^{\prime} \\
&+\frac{1}{4 \pi} \int_{S} \widehat{\mathbf{n}} \cdot \mathbf{M}\left(\mathbf{r}^{\prime}\right) \frac{\mathbf{r}-\mathbf{r}^{\prime}}{\left|\mathbf{r}-\mathbf{r}^{\prime}\right|^{3}} d^{2} r^{\prime}, \\
& \mathbf{H}_{\text {exchange }}(\mathbf{r})=\frac{2 A}{\mu_{0} M_{s}} \nabla^{2} \mathbf{m}(\mathbf{r}),
\end{aligned}
$$

where $\mathbf{m}=\mathbf{M} / M_{\mathrm{s}}$ is the normalized (unit) magnetization. In both cases, energy density $E=-(1 / 2) \mu_{0} \mathbf{M} \cdot \mathbf{H}$, where the $1 / 2$ factor arises from the dependence of $\mathbf{H}$ on $\mathbf{M}$. Thus the total energy density in the system is

$$
E_{\text {total }}=-\frac{\mu_{0}}{2} \mathbf{M} \cdot\left(\mathbf{H}_{\text {stray field }}+\mathbf{H}_{\text {exchange }}\right) .
$$

In soft films, the relevant length scale is the magnetostatic-exchange length, defined by

$$
\ell_{\mathrm{ex}}=\sqrt{\frac{A}{K_{\mathrm{d}}}},
$$




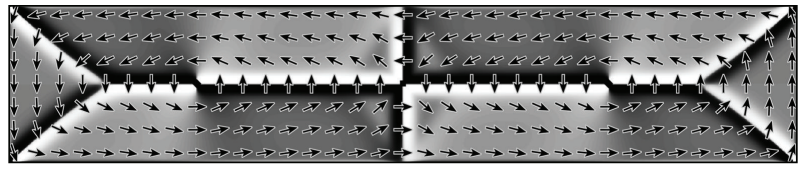

FIGURE 1: Zero-field equilibrium state from a micromagnetic simulation of a magnetically soft thin film rectangle with dimensions $500 \ell_{\mathrm{ex}} \times 100 \ell_{\mathrm{ex}} \times 6 \ell_{\mathrm{ex}}$. The shading indicates the magnetic charge - div $\mathbf{M}$, with black indicating negative charge and white positive charge.

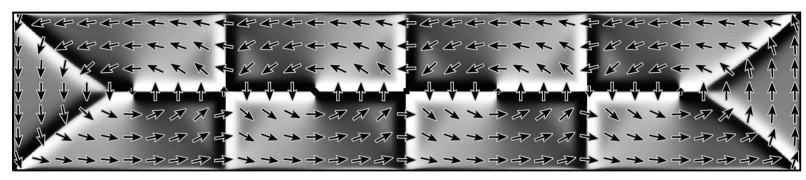

Figure 2: Simulation of the same system as in Figure 1, but in an equilibrium configuration featuring three cross-ties alternating with vortices; for this sample this is a lower energy state than the single cross-tie state in Figure 1.

where $K_{\mathrm{d}}$ is the magnetostatic energy density

$$
K_{\mathrm{d}}=\frac{1}{2} \mu_{0} M_{\mathrm{s}}^{2}
$$

All lengths reported herein are in units of $\ell_{\text {ex }}$, and energy densities are reported in units of $K_{\mathrm{d}}$. However, for the given values of $A$ and $M_{\mathrm{s}}, \ell_{\mathrm{ex}} \doteq 5.289 \mathrm{~nm}$ and $K_{\mathrm{d}} \doteq 464.7 \mathrm{~kJ} / \mathrm{m}^{3}$, so the results may be easily converted to $\mathrm{nm}$ and $\mathrm{J} / \mathrm{m}^{3}$ if desired.

\section{Simulation Details}

Aside from Figures 1 and 2, the simulations in this paper are periodic along the long axis of the wall, as indicated in Figure 3. This allows the structure of the cross-tie/vortex wall to be studied separately from the effects of edge domains. The period length is denoted by $X$, with $Y$ and $Z$ denoting the sample width and thickness, respectively. Coordinates are introduced such that positions $(x, y, z)$ inside the simulation volume run from $0 \leq x \leq X, 0 \leq y \leq Y$, and $0 \leq z \leq Z$.

The computational cells are approximately cubic in shape, with each edge dimension not larger than $1 / 2 \ell_{\text {ex }}$. This size is small enough that the maximum change in magnetization angle from one cell site to the next is kept below about $30^{\circ}$; this suffices to provide a good rendering of the magnetization on the discretized grid. The $y$ and $z$ cell sizes are adjusted downward if necessary to make the count of cells across each of the $y$ and $z$ dimensions odd, so that there is a unique center cell along each of those dimensions. The $x$ cell size is adjusted downward as necessary so that the count of cells along the $x$ dimension is $\equiv 2(\bmod 4)$ (so allowed cell counts are $2,6,10, \ldots)$. Taken together, these adjustments allow a unique cell in the center of each of the vortex and cross-tie cores to be identified.

The initial magnetization configuration for each simulation is either taken from the end state of a previous run (if one is available that is close to the dimensions of the

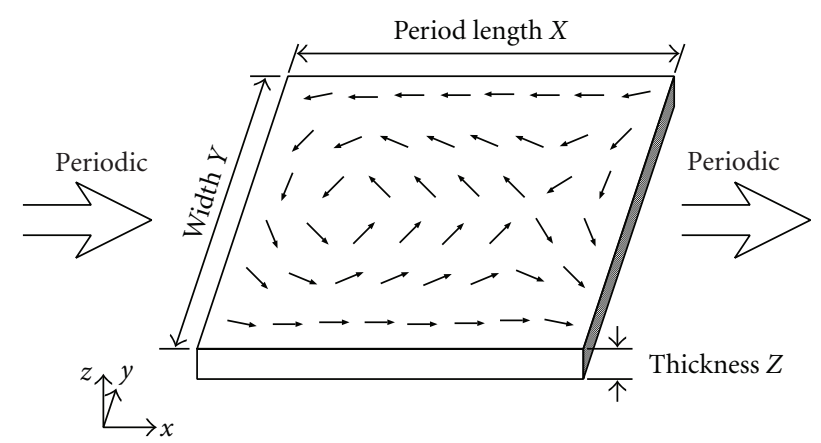

FIGURE 3: Simulations are computed on a rectangular volume representing a thin film strip with thickness $Z$, width $Y$, and an infinite length modeled by a periodic length of dimension $X$.

current run) or else set to a cartoon version of the crosstie/vortex configuration as illustrated in Figure 3. Either way, the magnetization in the central cell in the vortex core is set to $+z$ (along the film normal) and the central cell in the cross-tie core is set to $-z$. Except as noted, the magnetization in these center cells is held fixed. As discussed in Rave [8], this pinning tends to accelerate convergence of simulations to equilibrium and improves accuracy. Simulations run without this constraint show no discernible difference in the end equilibrium state. Some simulations were also run with the vortex and cross-tie cores both aligned in the $+z$ direction. This raises the energy somewhat, but for most geometries the energy difference is negligible, typically less than one part in $10^{5}$. For simulations with a period length to film thickness ratio of less that $10: 1$, however, the difference is larger. For example, in the $X=25 \ell_{\mathrm{ex}}, Y=200 \ell_{\mathrm{ex}}, Z=8 \ell_{\mathrm{ex}}$ case the energy in the aligned core setting was $3 \%$ larger than for the antialigned setting.

Once the initial magnetization is set, the simulation proceeds by energy minimization via a conjugate-gradient procedure, stopping when the reduced torque $|m \times H| / M_{\mathrm{s}}<$ $1.2 \times 10^{-8}$.

\section{Results and Discussion}

Each point in Figure 4 marks the average total energy density at equilibrium resulting from a simulation with width $Y=$ $200 \ell_{\text {ex }}$ at the indicated thickness $Z$ and period length $X$. There is one cross-tie/vortex pair in each period, so as the period length $X$ grows large the wall becomes primarily two Néel segments interrupted by a cross-tie and vortex at either end. This is evident in the behavior of the curves for large $X$, as for each thickness $Z$ the curve asymptotically converges to the energy density of the associated Néel wall. For small period lengths $\left(X<100 \ell_{\mathrm{ex}}\right)$ the energy density grows sharply as the exchange energy resists compression of the complex cross-tie/vortex structure. For thinner films ( $Z$ under about $1 \ell_{\text {ex }}$ for $Y=200 \ell_{\text {ex }}$ ), the energy density curves are monotonically decreasing. This means that in a thin infinite strip if the cross-tie and vortex are not pinned by some means, they will spread out indefinitely leaving behind a plain Néel wall. This is consistent with the experimental 


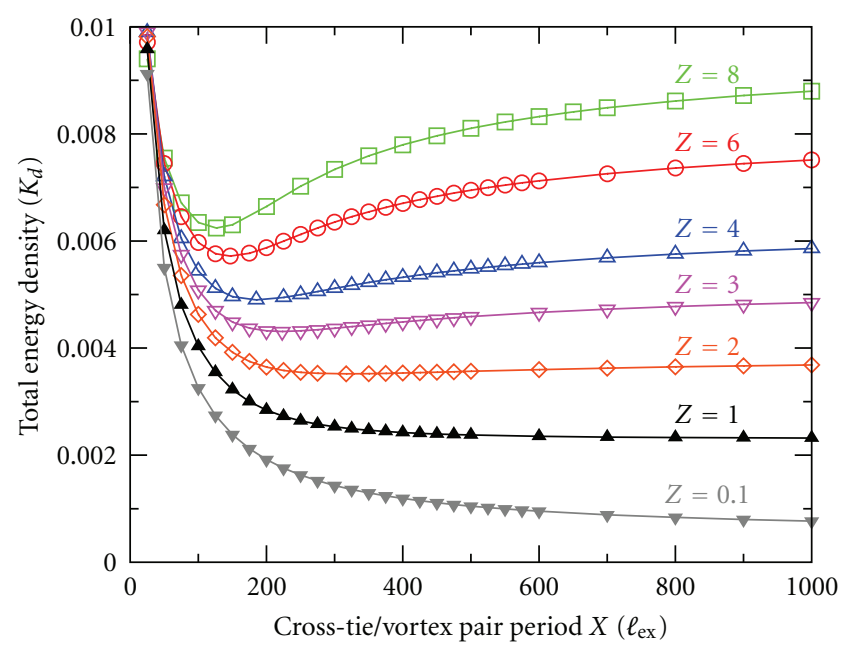

FIGURE 4: Average total energy density as a function of period length $X$ for simulations with width $Y=200 \ell_{\mathrm{ex}}$ for various thicknesses $Z$ (measured in $\ell_{\mathrm{ex}}$ ). Symbols represent simulation results, lines are a guide to the eye. Each of the curves with $Z \geq 2 \ell_{\mathrm{ex}}$ exhibits a minimum value between $125 \ell_{\mathrm{ex}}$ and $320 \ell_{\mathrm{ex}}$. In this range, the curves for $Z \leq 1 \ell_{\text {ex }}$ are monotonically decreasing with $X$.

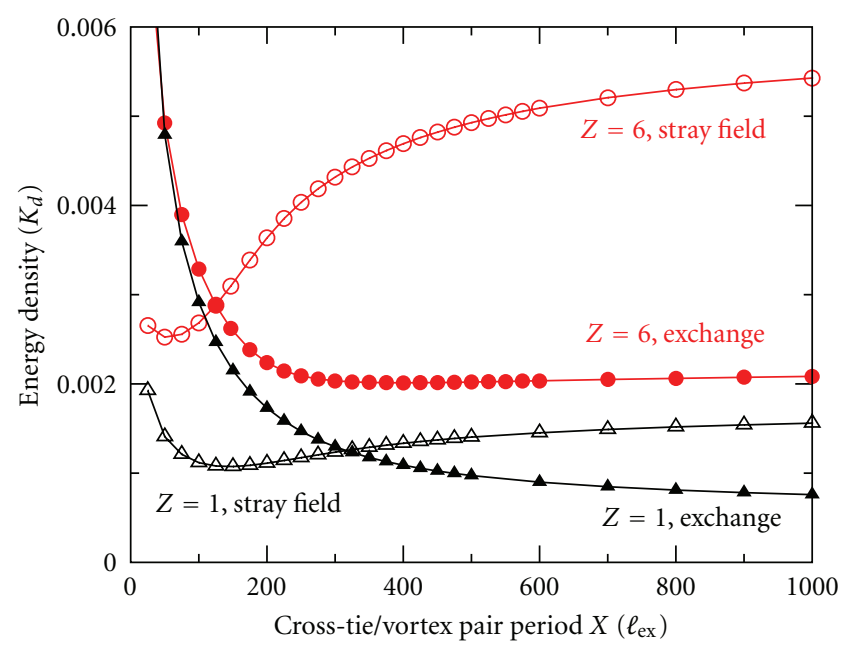

FIGURe 5: Average stray field (open symbols) and exchange energy (filled symbols) densities as a function of period length $X$ for width $Y=200 \ell_{\mathrm{ex}}$ at thicknesses $Z=6 \ell_{\mathrm{ex}}$ and $1 \ell_{\mathrm{ex}}$. These curves are a decomposition of the corresponding total energy density curves from Figure 4 into constitutive parts.

result that cross-tie/vortex walls are not observed in ultrathin films. The transition thickness is a function of the strip width $Y$; this dependence is explored in Figure 9 below.

For thicker films there is a unique minimum on each curve, which corresponds to a "natural" period length $X$ this is the period length that minimizes the energy for a crosstie/vortex wall at the given film thickness and strip width. Note though that each point in Figure 4 is a stable equilibrium configuration under the fixed period assumption. So if the period length is constrained by geometry or other means such as pinning defects, then period lengths other than the natural length are possible, as seen in Figures 1 and 2.

The energy wells are asymmetric, especially for the midlevel thicknesses, say $Z$ between $2 \ell_{\mathrm{ex}}$ and $4 \ell_{\mathrm{ex}}$. In this regime the penalty for a period longer than the natural period is small, but in all cases periods significantly shorter than the natural period are energetically prohibitive.

Another feature of the curves in Figure 4 is that as the films grow thicker, the energy density increases, the natural period length grows shorter, and the energy well is deeper. These effects can be explained by examining the stray field and exchange component energies of the total energy.

In this regard, note two attributes of the magnetization in these simulations. The first is that the shape anisotropy of thin films constrains the magnetization to lie mostly in-plane (the notable exception being of course the cores of vortex and cross-tie structures). The second is that the magnetization does not vary much along the film normal $(z)$ direction. This is due in part to the dominance of exchange coupling over the relatively short distance between the top and bottom of each film and also due to the relative uniformity of stray field in $z$. (The latter condition does not hold near the vicinity of the vortex and cross-tie cores, and this leads to nearby $z$ variation in $\mathbf{m}$, as will be seen below.)

The second attribute means we can meaningfully consider a situation where the magnetization is held fixed and the thickness of the part is varied. In this setting the first integral in the formula for the stray field (1), which handles the effects of the bulk charge, is seen to vary linearly with thickness $Z$ via the change in the part volume. Ignoring magnetic charges on the top and bottom surfaces in the second integral, we see that it too varies linearly with $Z$ (although in the Landau flux-closure structures considered here this contribution is minor regardless). The net result is that if the magnetization were held fixed, then reducing the film thickness would be expected to reduce the stray field by a similar amount. The exchange field (2), however, does not vary with thickness $Z$. This means that one can expect exchange to take on a more dominant role as the film thickness is decreased.

These effects are on display in Figure 5, which breaks down the total energy density curves from Figure 4 for two thicknesses into the stray field and exchange components. For both thicknesses we see the dominance of the exchange energy in short-period lengths, giving way to the stray field energy for longer period lengths. Moreover, for each component the energy density is greatly reduced in the thinner strip. If the magnetization configurations were the same for the two thicknesses, then by the above analysis the exchange energy density would stay constant and the stray field energy density would drop by a factor of six. In practice, of course, what happens is that the weak stray field in the thinner film allows the magnetic structures to expand, reducing the exchange energy at the expense of a modest increase in the stray field.

To understand how cross-tie/vortex structures lower the energy density of a Néel wall, return to Figure 1, and focus first on a section of the Néel portion of the wall between the left hand vortex and the cross-tie. Moving from bottom to 


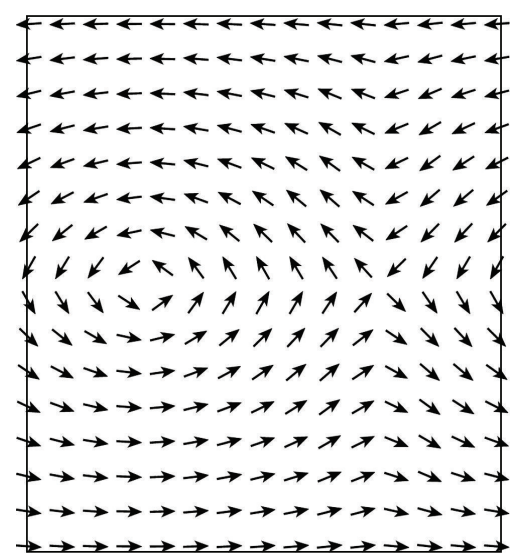

(a)

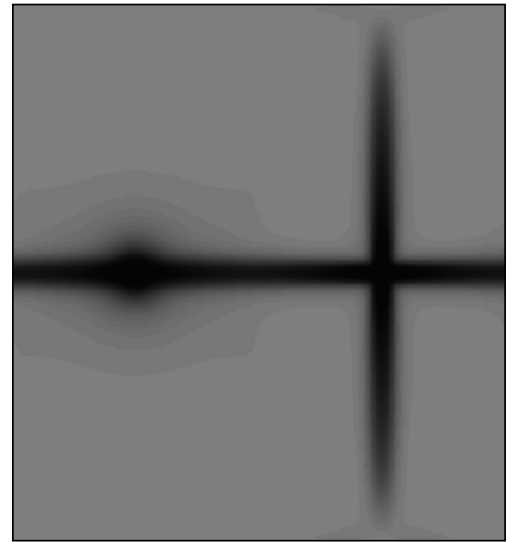

(c)

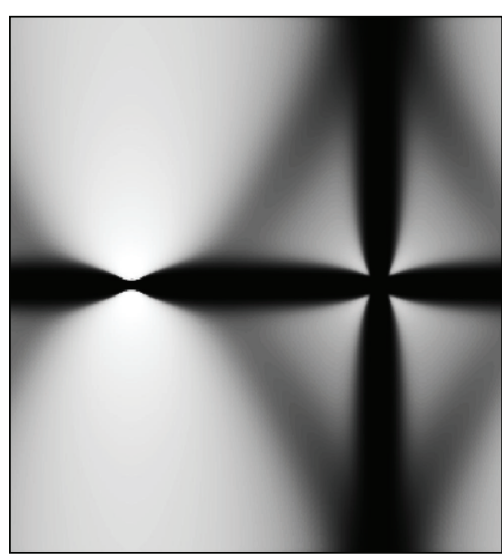

(b)

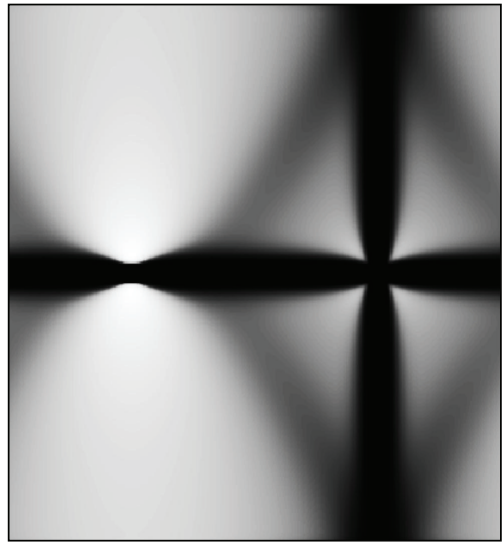

(d)

Figure 6: Equilibrium configuration for width $Y=200 \ell_{\mathrm{ex}}$, thickness $Z=4 \ell_{\mathrm{ex}}$, and $X$-period length $185 \ell_{\mathrm{ex}}$ (which is the natural period length for this $Y$ and $Z$ ). (a) Magnetization pattern, (b) stray-field energy density, (c) exchange energy density, and (d) total energy density. In (b)-(d), the shading scale runs from white (low energy density) to black (high energy density).

top across the wall, the magnetization rotates counterclockwise. This configuration produces negative magnetic charge on the lower half of the strip (dark region) and positive charges on the upper half (light region). (Here "lower" and "upper" refer to the view on the page.) This sets up a stray field running from the positive charges to the negative charges, counter to the magnetization in the center of the wall, making the wall center a high stray field energy density region. On the right hand side of the cross-tie the magnetization rotation direction across the wall is reversed, so that the positive charge region is below the wall and the negative charge region is above. The wall is still a high stray field energy density region, but the checkerboarding of the charge regions reduces the total stray field energy in two ways. The first factor is that by effectively arranging the charge regions into a quadrupole configuration, the extent of their stray field is reduced. The second, larger contribution is seen more clearly in Figure 2. In the checkerboard pattern, stray field between the charge blocks runs not only up and down across the Néel sections of the wall, but also left and right horizontally parallel to the wall. The orientation of the magnetization about each vortex core is such that it aligns with the stray field from the nearby charge blocks, so that the regions above and below each vortex are regions of low stray field energy density.

This latter effect is shown directly in Figure 6, which is from the (periodic) simulation corresponding to the minimum point on the $Z=4 \ell_{\text {ex }}$ curve in Figure 4 . Parts (b)-(d) of this figure are shaded to indicate the component and total energy densities as a function of position. In part (b), the light-colored low energy density regions above and below the vortex core are clearly visible. Part (c) shows the regions of high exchange energy density. These include the vortex core and center of the wall, as expected. It is interesting that there is also a region of high exchange energy running perpendicular to the wall through the cross-tie core. In an idealized cross-tie, the magnetization rotates around the cross-tie core in the same manner as the magnetization rotates about a vortex core, only with the opposite winding number. In such a configuration the exchange energy is exactly the same as for a vortex (the stray field energy is a different matter, of course), so the high exchange energy spike perpendicular to the wall must arise due to deformation of the cross-tie structure. Most likely the observed buckling in the magnetization along this line is caused by the horizontal stray field from the checkerboard 


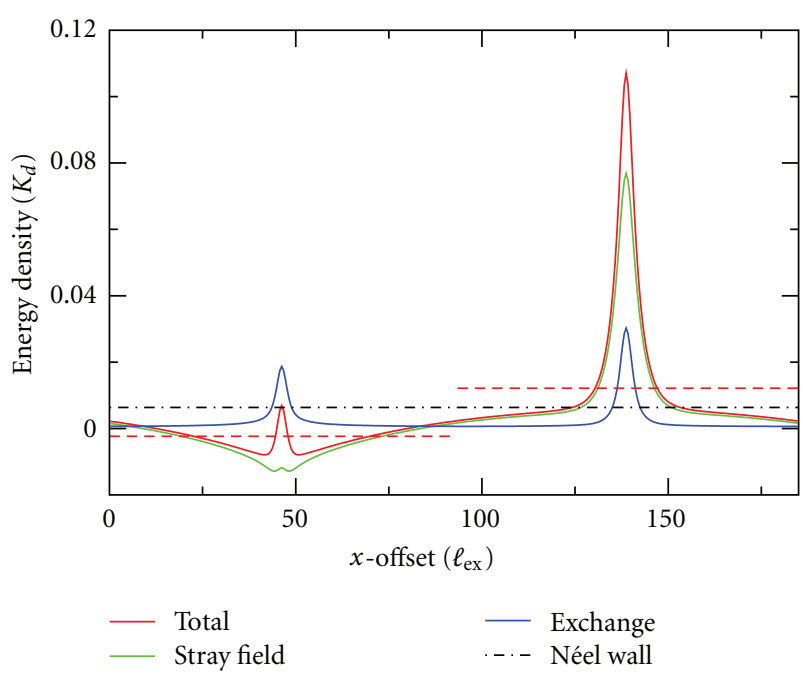

Figure 7: Cross-sectional $(y z)$ averages for total, stray field, and exchange energy densities as a function of the $x$ location along the wall for the simulation in Figure 6, and the energy density for a Néel wall in the same geometry. The center of the vortex core is in the cross-section at offset $x=46 \ell_{\mathrm{ex}}$, and the center of the cross-tie core is at $x=139 \ell_{\mathrm{ex}}$. The two half-width dashed red lines indicate the average energy density of the cross-tie/vortex wall across each corresponding half-period.

charge regions which flows counter to the magnetization here.

Additional details may be gleaned from Figure 7, which shows cross-sectional averages for Figure 6 and includes a black-dashed line showing the energy density for a pure Néel wall in this geometry. The energy savings in stray field energy around the vortex core, and expense around the crosstie core, are shown by the green line. On either side of the cross-tie and vortex cores, the magnetization spreads out slightly as compared to a Néel wall, and as a result the exchange energy density (blue line) in those regions is slightly less than the exchange energy density for a Néel wall (not shown). This savings is more than offset by the increase in exchange energy inside the vortex and cross-tie core structures, so that in total the exchange energy for the cross-tie/vortex wall is higher than the exchange energy for the Néel wall. (Another view of this is that the wall structure outside the cores is essentially that of a stretched Néel wall; the stretching reduces the exchange energy, but the stray field energy across the wall is increased by more than the exchange energy reduction.) The dashed red line shows the combined (stray field plus exchange) energy density averaged across each half of the simulation volume. This shows a clear reduction in energy density as compared to the Néel wall for the portion of the simulation about the vortex, and a clear increase about the cross-tie. The average of these two halflines is the average energy density for the cross-tie/vortex wall as a whole, which is slightly below the energy density for the pure Néel wall. An important point here is that the cross-tie structure by itself costs energy as compared to the Néel wall; the cross-tie/vortex wall formation as a whole is

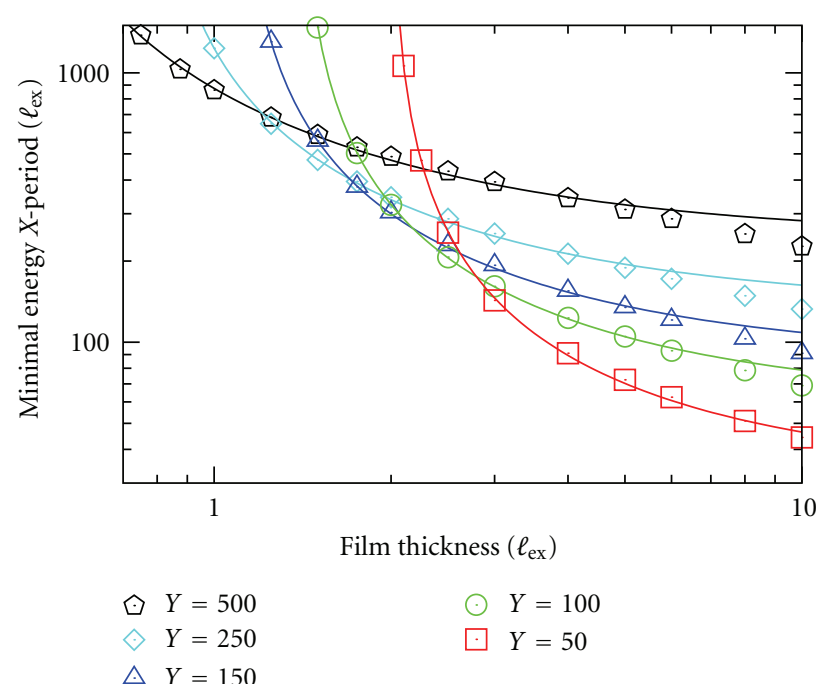

FIGURE 8: Cross-tie/vortex pair period length $(X)$ having the lowest energy density as a function of film thickness $(Z)$ for five strip widths $\left(Y\right.$, in $\left.\ell_{\text {ex }}\right)$, as labeled (log-log scale). Symbols show data from micromagnetic simulations; curves are least-square fits through data to the functional form $X=A /((Z / B)-1)+C$; the corresponding values for $A, B$, and $C$ for each curve are given in Table 1.

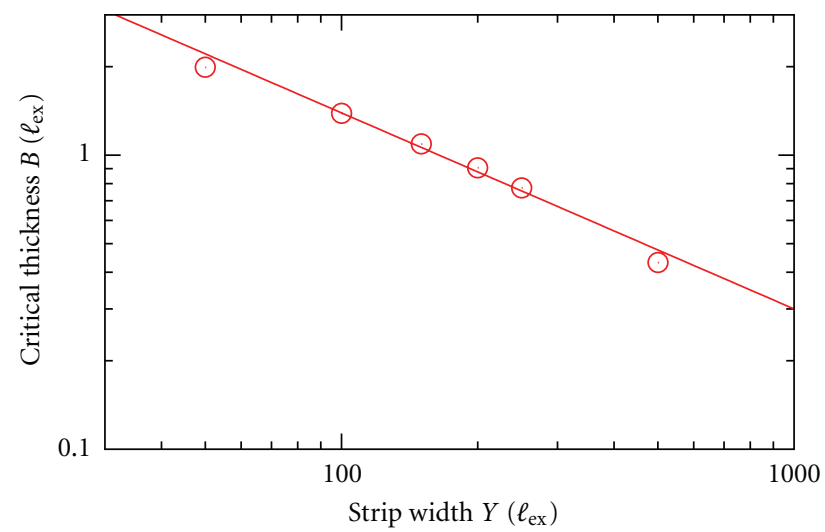

FIGURE 9: Critical thickness at which the Néel wall has lower energy density than a cross-tie/vortex wall of period $X$, for all $X$, as a function of strip width $Y$. Data points are from Table 1, the line is the fit curve $30 / Y^{2 / 3}$.

energetically favorable only because of the stray field energy savings associated with the vortex structure.

The two graphs, Figures 8 and 9, collect information on the natural period lengths from Figure 4 and similar simulation series for several other strip widths. For each strip width $Y$ and thickness $Z$, a sequence of simulations was performed using a golden section search to locate the precise $X$-period that minimized the total energy density. In Figure 8 we observe that for each strip width $Y$, the minimal $X$-period length data can be fitted fairly well by a curve of the form $X=A /((Z / B)-1)+C$, where $Z$ is the film thickness and $A, B$, and $C$ are fit parameters. In this fit only the data for $Z<8 \ell_{\text {ex }}$ are used; in the thicker films the cross-tie and vortex 


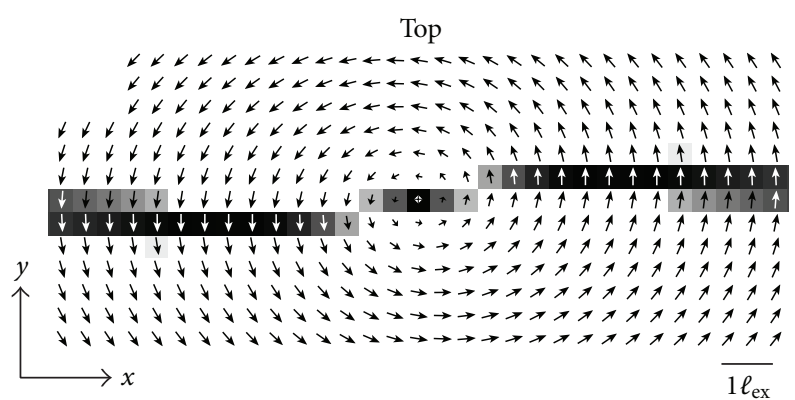

(a)

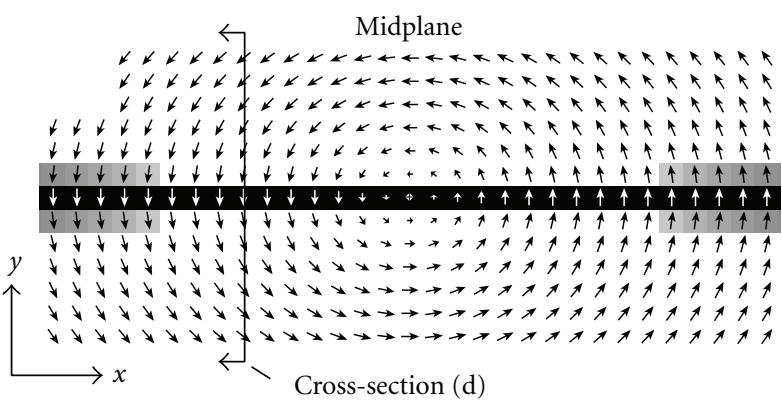

(b)

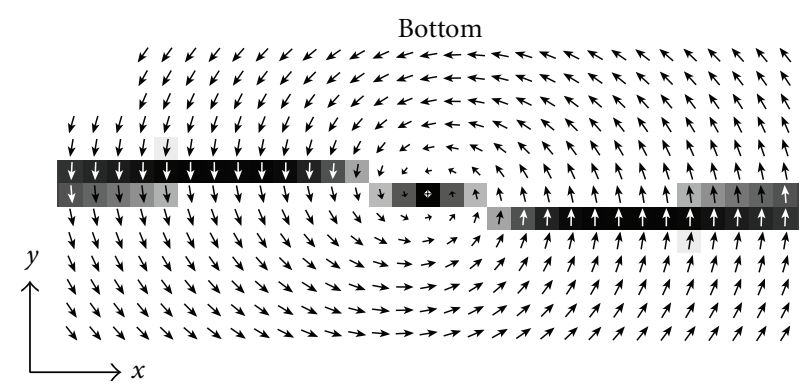

(c)

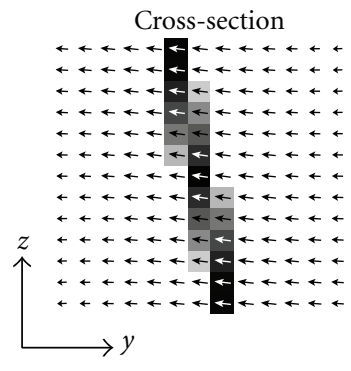

(d)

FiguRE 10: Enlarged view of a $15 \ell_{\mathrm{ex}} \times 6 \ell_{\mathrm{ex}}$ subsection about a vortex in a simulation having $X$-period length of $147 \ell_{\mathrm{ex}}$, width $Y=200 \ell_{\mathrm{ex}}$, and thickness $Z=6 \ell_{\mathrm{ex}}$. Parts (a), (b), and (c) show the top, middle, and bottom planes, respectively, while (d) is a cross-section through the full thickness of the sample at the location marked in (b), roughly $3.5 \ell_{\mathrm{ex}}$ to the left of the vortex core. The shading indicates the absolute value of the $x$-component of the magnetization, with black at $m_{x}=0$.

Top

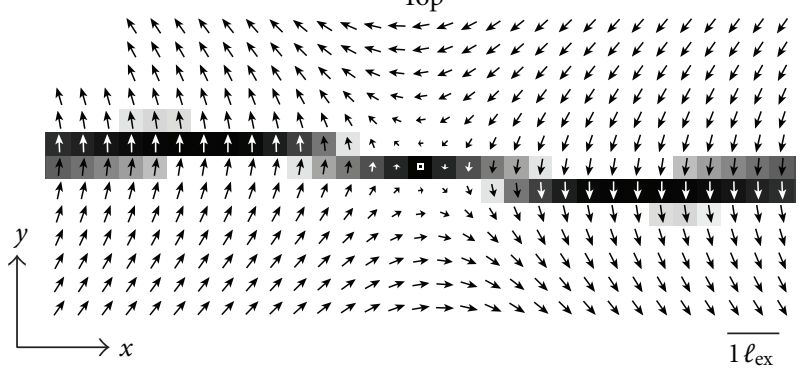

(a)

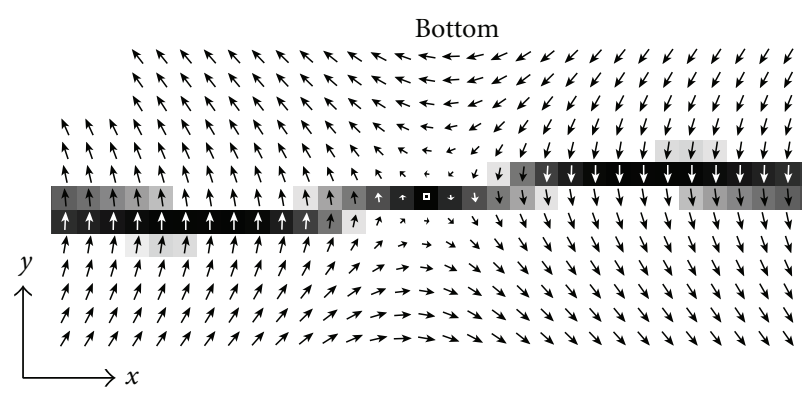

(c)

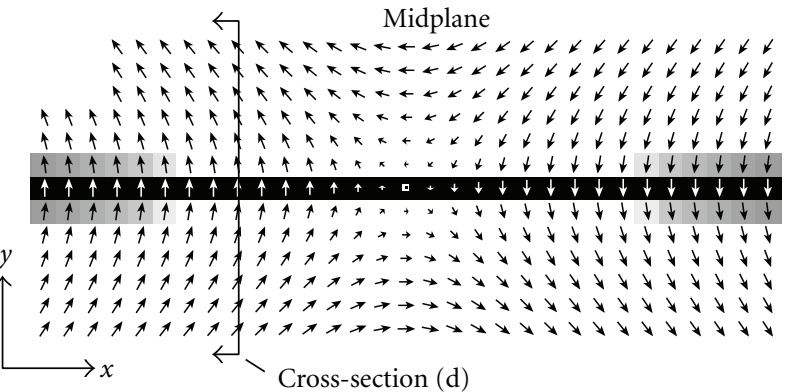

(b)

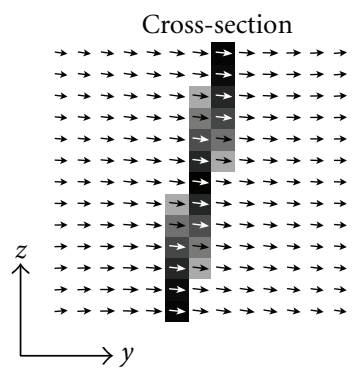

(d)

Figure 11: Companion image to Figure 10, this enlarged view of a $15 \ell_{\mathrm{ex}} \times 6 \ell_{\mathrm{ex}}$ subsection is about the cross-tie in the same simulation with $X$-period length of $147 \ell_{\mathrm{ex}}$, width $Y=200 \ell_{\mathrm{ex}}$, and thickness $Z=6 \ell_{\mathrm{ex}}$. Parts (a), (b), and (c) show the top, middle, and bottom planes, respectively, while (d) is a cross-section through the full thickness of the sample at the location marked in (b), roughly $3.5 \ell_{\mathrm{ex}}$ to the left of the cross-tie core. The shading indicates the absolute value of the $x$-component of the magnetization, with black at $m_{x}=0$. 
TABLE 1: Coefficients to functional form $X=A /((Z / B)-1)+C$ for various strip widths $Y$ to fit simulation results for minimal energy $X$-period (see Figure 8).

\begin{tabular}{lccc}
\hline $\begin{array}{l}\text { Strip width } \\
\left(\ell_{\mathrm{ex}}\right)\end{array}$ & $\begin{array}{c}A \\
\left(\ell_{\mathrm{ex}}\right)\end{array}$ & $\begin{array}{c}B \\
\left(\ell_{\mathrm{ex}}\right)\end{array}$ & $\begin{array}{c}C \\
\left(\ell_{\mathrm{ex}}\right)\end{array}$ \\
\hline 50 & 58 & 1.988 & 32 \\
100 & 117 & 1.386 & 60 \\
150 & 177 & 1.091 & 87 \\
200 & 243 & 0.906 & 113 \\
250 & 320 & 0.774 & 136 \\
500 & 832 & 0.431 & 245 \\
\hline
\end{tabular}

structures develop significant asymmetry which alters the character of the structure. If this asymmetry did not develop, then the parameter $C$ would describe the asymptotic period length that would be obtained in thick films.

At the other end of the scale, we see that each of the curves in Figure 8 has a pole on the left, which corresponds to parameter $B$. As discussed above with respect to the $Z=0.1 \ell_{\mathrm{ex}}$ curve in Figure 4, if a film is thin enough then there is no minimal period length. For each strip width $Y$, the critical thickness dividing the two regimes corresponds to the location of the pole in Figure 8 (or, equivalently, parameter $B$ ). The critical thickness as a function of strip width $Y$ is plotted on a log-log scale in Figure 9. We see from the fitted curve, $Z_{\text {crit }} \approx 30 / Y^{2 / 3}$, that the critical thickness decreases with increasing strip width. From a practical standpoint the natural period length can be quite large. For example, although the $Z=1 \ell_{\mathrm{ex}}$ curve in Figure 4 appears to be monotonically decreasing, the fit in Table 1 predicts a minimum at $X=2455 \ell_{\mathrm{ex}}$, or roughly $13 \mu \mathrm{m}$ in NiFe.

As an example of the use of Figure 8, refer again to the finite system of Figures 1 and 2 . If we allow for a $50 \ell_{\text {ex }}$ border at each of the left and right sides of the sample to accommodate edge closure domains, then that leaves a $400 \ell_{\mathrm{ex}}$ run in the middle for the cross-tie/vortex wall. From Figure 8, we see that the natural period length for a strip of width $100 \ell_{\mathrm{ex}}$ and thickness $6 \ell_{\mathrm{ex}}$ is just under $100 \ell_{\text {ex }}$. This predicts that a configuration with four crosstie/vortex periods would have lower energy than either the three period configuration shown in Figure 2 or a five period configuration. Direct simulations on the finite system bear out this result.

In regions where the magnetization lies in-plane, the stray field is nearly uniform through the thickness of the film, and so the magnetization also shows little variation in $z$. The vortex and cross-tie core regions, however, are delineated by out-of-plane magnetization, and this does produce a $z$-dependence on the magnetization near the cores. This effect is shown in Figures 10 and 11. (For this simulation, the magnetization in the central cells of the cores was not pinned.) Looking first at the magnetization at the top surface around the vortex core (Figure 10(a)), the magnetization in the vortex core points out of the plane, and the resulting positive magnetic charge interacts with the checkerboard magnetic charge pattern on either side by pushing the positive charge blocks away (towards $-y$ on the left and $+y$ on the right) and extending the negative charge regions. This causes a "micro-deformation" of the wall [7]; the spacing between the arrows in the diagram is roughly $1 / 2 \ell_{\text {ex }}$, so the total deformation is about $1 \ell_{\text {ex }}$. Outside the viewed region, at about $12 \ell_{\mathrm{ex}}$ on either side of the core, the wall shifts back to the center line.

On the bottom surface of the film (Figure 10(c)), the surface charge from the core has the opposite charge, and the wall shifts in the opposite direction, while the midplane shows no shift at all (Figure 10(b)). A cross-section through the thickness of the film (Figure 10(d)) shows that the wall is actually canted by about $10^{\circ}$ from the vertical. Outside of this view area, the Néel portions of the wall are not canted, but run through the thickness of the film independent of $z$. Figure 11 shows that the magnetization around the cross-tie core behaves similarly.

This effect relies on the opposite charges on the top and bottom surfaces being sufficiently far apart that they can independently influence the nearby magnetization. For thinner films, the extent of the dipole field from the core diminishes relative to the exchange length and the wall canting is reduced; for films thinner than about $1 \ell_{\mathrm{ex}}$ the effect is not evident.

\section{Conclusion}

In an ideally soft magnetic thin film, the $180^{\circ}$ cross-tie/vortex wall is a periodic structure consisting of alternating crossties and vortices sandwiched between Néel wall segments having alternating chiralities. This structure is primarily two dimensional (i.e., independent of $z$ ), with the exception of minor canting on either side of both cross-tie and vortex cores in films thicker than $1 \ell_{\mathrm{ex}}$.

In an infinite strip, the cross-tie/vortex wall structure has a natural period length that minimizes the energy density for this class of walls, and this length is a function of both the strip thickness and width. For a given strip width $Y$, there is a critical thickness $Z_{\text {crit }}$ below which the natural period length is infinite. That relation is $Z_{\text {crit }} \approx 30 / Y^{2 / 3}$, with $Z_{\text {crit }}$ and $Y$ measured in $\ell_{\text {ex }}$. In films thicker than $Z_{\text {crit }}$, the energy reduction obtained by a cross-tie/vortex wall as compared to a plain Néel wall is the result of decreased stray field energy across the outboard sides of the vortex cores arising from the checkerboarding of the magnetic charge associated with the Néel wall segments.

Related periodic wall structures are topologically permissible, for example one could replace each vortex in the cross-tie/vortex structure with a counterrotating cross-tie, or replace each cross-tie with a counterrotating vortex. The former is probably energetically ill-favored, but the latter produces the well-known diamond state that frequently sports lower energy than the cross-tie/vortex wall [8].

\section{References}

[1] R. Kirchner and W. Döring, "Thin films: domain walls and related topics," Journal of Applied Physics, vol. 39, pp. 855-856, 1968. 
[2] D. V. Berkov, K. Ramstöck, and A. Hubert, "Solving micromagnetic problems. Towards an optimal numerical method," Physica Status Solidi, vol. 137, pp. 207-225, 1993.

[3] K. Ramstöck, W. Hartung, and A. Hubert, "The phase diagram of domain walls in narrow magnetic strips," Physica Status Solidi, vol. 155, no. 2, pp. 505-518, 1996.

[4] R. Ploessl, J. N. Chapman, A. M. Thompson, J. Zweck, and H. Hoffmann, "Investigation of the micromagnetic structure of cross-tie walls in permalloy," Journal of Applied Physics, vol. 73, no. 5, pp. 2447-2452, 1993.

[5] A. Hubert and R. Schäfer, Magnetic Domains, Springer, 1998.

[6] S. Hou, G. Pan, D. J. Mapps et al., "Magnetic force microscopy and micromagnetic study of cross-tie wall structures in $\mathrm{Co}_{91} \mathrm{Nb}_{6} \mathrm{Zr}_{3}$ amorphous thin films," Journal of Applied Physics, vol. 87, no. 3, pp. 1096-1102, 2000.

[7] M. Redjdal, A. Kakay, M. F. Ruane, and F. B. Humphrey, "Cross-tie walls in thin permalloy films," IEEE Transactions on Magnetics, vol. 38, no. 5, pp. 2471-2473, 2002.

[8] W. Rave and A. Hubert, "Magnetic ground state of a thin-film element," IEEE Transactions on Magnetics, vol. 36, no. 6, pp. 3886-3899, 2000.

[9] R. Hertel, "Thickness dependence of magnetization structures in thin Permalloy rectangles," Zeitschrift für Metallkunde, vol. 93, no. 10, pp. 957-962, 2002.

[10] F. Alouges, T. Rivière, and S. Serfaty, "Néel and cross-tie wall energies for planar micromagnetic configurations," ESAIM, vol. 8, pp. 31-68, 2002.

[11] A. DeSimone, H. Knüpfer, and F. Otto, "2-d stability of the Néel wall," Calculus of Variations and Partial Differential Equations, vol. 27, no. 2, pp. 233-253, 2006.

[12] M. J. Donahue and D. G. Porter, "OOMMF user's guide, version 1.0," NISTIR 6376, National Institute of Standards and Technology, Gaithersburg, Md, USA, 1999. 

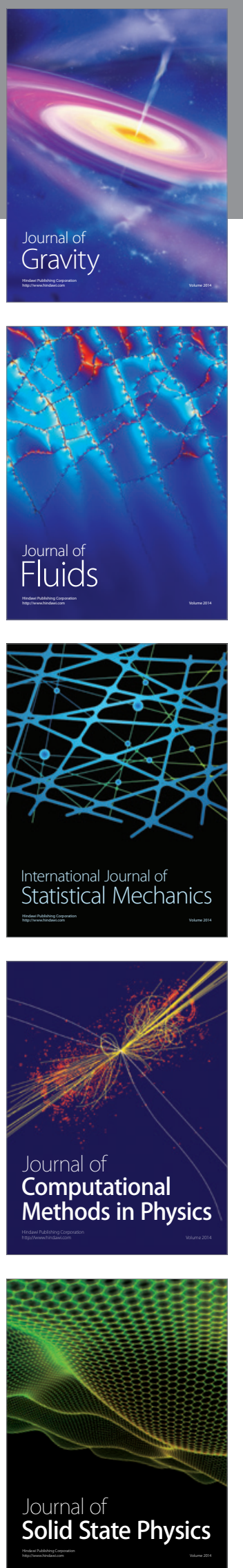

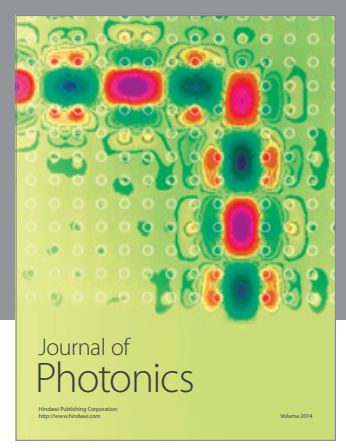

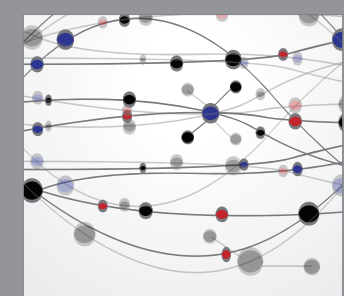

The Scientific World Journal
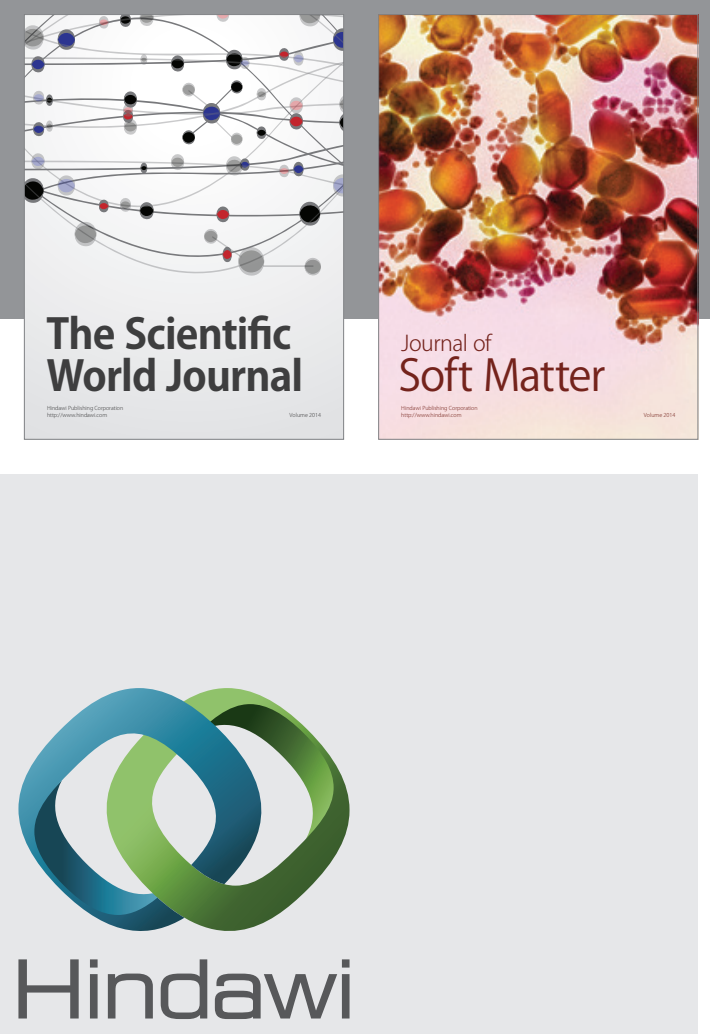

Submit your manuscripts at

http://www.hindawi.com
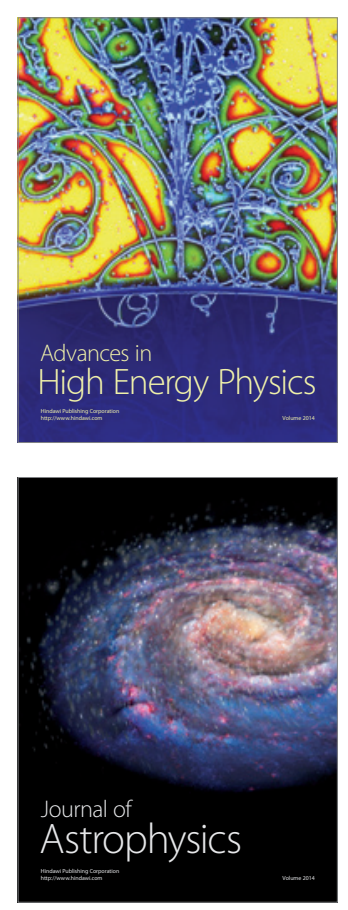
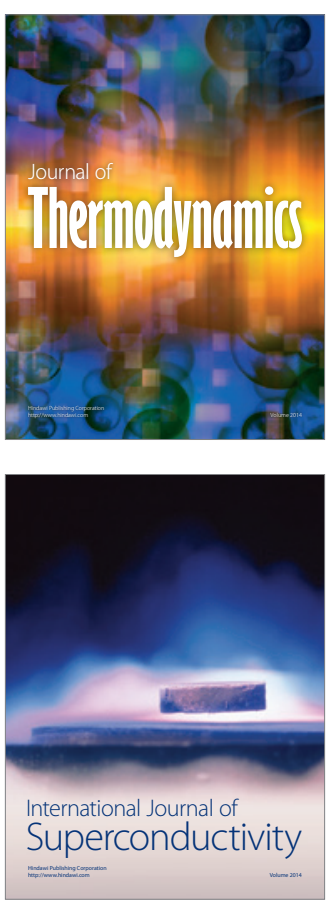
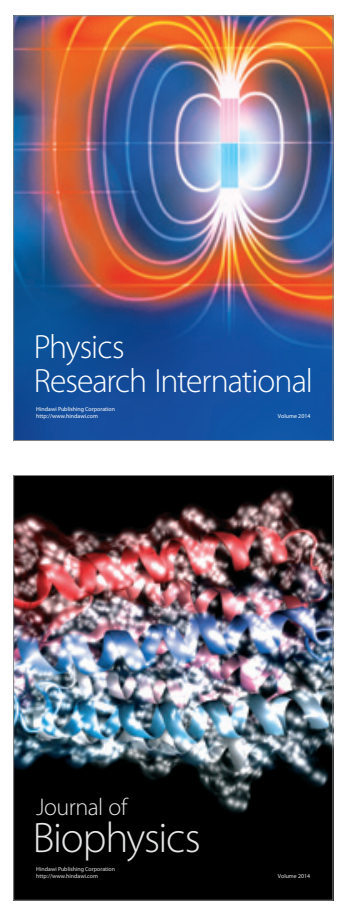
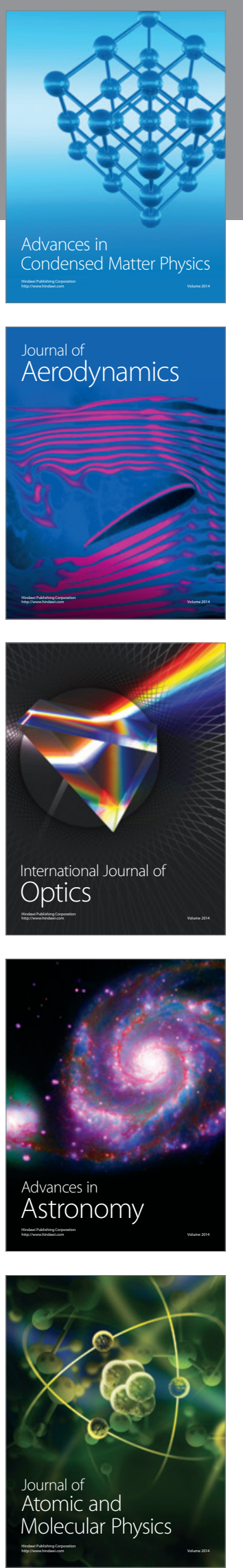